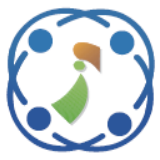

\title{
Fusion Based Automatic Human Age Estimation Using Local Descriptors
}

\author{
Penubaka Kiran Kumar Reddy ${ }^{1 *}$ \\ ${ }^{1}$ Malla Reddy Institute of Engineering and Technology-Jawaharlal Nehru Technological University Hyderabad, \\ Department of Computer Science \& Engineering, Hyderabad, India \\ * Corresponding author's Email: kiran.penubaka@gmail.com
}

\begin{abstract}
This paper introduces a new age estimation method based on the fusion of local features extracted using histogram-based local texture descriptors. In this study, the age estimation performances of well-known powerful texture descriptor with improved modification Local Binary Patterns (mLBP), and Local Phase Quantization (LPQ), which have not been analysed in depth for age estimation, investigated. In addition, multi-scale and spatial texture analysis performed for all descriptors. In the spatial texture analysis, a new approach using Overlapped Blocks obtained by discrete blocks proposed to reduce the overlapped age groups between the neighbour age group. Then feature fusion performed to investigate the age estimation accuracies of different combinations of local texture descriptors. Multiple Linear Regression (MLR) used to estimate the specific age at the end of this proposed system. The results show that the age estimation accuracy of the proposed method is better when compared to previous methods on FG-NET, MORPH databases.
\end{abstract}

Keywords: Age estimation, Age classification, Multilevel LBP, FG-NET, MORPH.

\section{Introduction}

Age estimation is the process of associating a facial image automatically with an exact age or age group. In order to facilitate age estimation, suitable facial representations are necessary; as the most robust classifiers will fail due to the inadequacy of the domain where the feature recognition is done [1]. Hence, the design of age estimation systems requires careful selection of the aging facial features. However, the facial image characteristics make this problem very difficult to solve. The varying illumination, background, pose, expression of the subject are the common problems of facial image processing systems. Furthermore, real world age progression displayed on faces is uncontrollable and personalized. In other words, the variation on different individual's face is not the same during their lifespan. It depends on the factors such as genetics, race, living styles, eating and drinking habits, climate, etc. [2]. Exposure to sunlight, smoking, extreme weight loss, emotional stress, extent and frequency of facial expressions, usage of anti-aging products, and plastic surgery also affect the age estimation performance [3]. Another challenge in age estimation systems is the availability of a good aging database. As aging is a slow process, a large aging database, especially containing the chronometrically image series of individuals is hard to collect.

In this paper, a new age estimation method with the effects of different histogram based local texture descriptors introduced. The idea of using texture information of facial images is not new. However, improved multilevel LBP (mLBP) and Local Phase Quantization (LPQ) utilized to analyse age estimation on FG-NET, MORPH and PAL databases [4]. In our method, the original facial images normalized so that the orientation and the size of faces adjusted and only the facial region extracted. After that Multilevel Local Binary Patterns (mLBP) and LPQ descriptors are used to extract the age related features from normalized facial images. In mLBP extraction, a new approach used to reduce the Overlapped Block (OB) age group clash. In addition, multi scale analysis performed for holistic, spatial and OB based LPQ and mLBP texture descriptors for 
finding the best estimation accuracy. Then those feature vectors, which give the best estimation accuracies, fused in feature level to investigate the age estimation performances of different combinations of local texture descriptors. Multiple Linear Regression (MLR) used to estimate the specific age.

This paper organized as follows. Some of the well-known age estimation techniques are analysed in Section 2. The proposed Fusion based age estimation approach explained in Section 3. In Section 4 the experimental results on various databases are reported and analysed. Finally, the conclusions outlined in Section 5.

\section{Literature review}

Researchers in age estimation suggested several research techniques. A brief evaluation of some essential contributions to the existing literatures presented in this section.

D. Lai, Y. Chen, X. Luo, J. Du, and T. Wang [5] proposed a new method for estimating the age of facial image into a dynamic range or a discrete age set rather than a single age or age group. A new measurement was introduced, i.e. Confidence Interval/Confidence level to evaluate the performance of proposed method. In addition, presented the result of estimated age for a facial image should be a dynamic range instead of a fixed age or fixed group predefined. Through, many experiments and observations, the existing method found that the estimated ages of facial images with the same ground-true age are normal distribution with mean value is close to ground-true age. The proposed technique was less suitable for recognizing the inclined facial images, because it took high response time and identification rate.

M. Kilinc, and Y.S. Akgul, [6] have presented an age estimation system that uses the fusion of geometric features (ratios of distance values between facial landmark points) and textural features (filter responses of the face image pixel values). First, a group of classifiers calculated the probabilities of a face image belonging to each overlapping age groups. Then an interpolation-based technique used to produce the final estimated age. Many different textural features and geometric features compared in this study. The results of the experiments showed that the fusion with the geometric features increases the performance of the textural features and the highest age estimation rates obtained using the fusion of Local Gabor Binary Patterns and Geometric features with overlapping age groups. While using a large number of features, a perceivable over-fitting of the model observed that decreases the accuracy of the training set.

Y. Li, Z. Peng, D. Liang, H. Chang, and Z. Cai, [7] have propose a novel hierarchical feature composition and selection model used in facial age estimation. Firstly, select the features in a boosting way and then weightily combined adjacent selected features. The whole process of feature selection and combination named as a boosting layer. Then, multiple boosting layers were stacked into a hierarchical model. In each boosting layer, a number of weak classifiers comprised the selected features, and their combination weights were inversely proportional against the training errors of weak classifiers. The experimental results on two aging face databases MORPH and FG-NET had shown significant reduction on mean absolute error of age estimation compared with other state-of-the-art methods. In a few cases, the training data were supervised evaluation or manual adjustment, which needed to automate.

J. Lu, V.E. Liong, and J. Zhou [8] proposed a costsensitive local binary feature learning (CS-LBFL) method for facial age estimation. It learns a series of hashing functions to project raw pixel values extracted from face patches into low-dimensional binary codes, where binary codes with similar chronological ages were projected as close as possible, and those with dissimilar chronological ages were projected as far as possible. Then, pool and encode these local binary codes within each face image as a real-valued histogram feature for face representation. In addition, a cost-sensitive local binary multi-feature learning method was proposed to jointly learn multiple sets of hashing functions using face patches extracted from different scales to exploit complementary information. The proposed method achieved competitive performance on four widely used face-aging data sets. While performing with semi-supervised approach (CS-LBFL), the semantic gap maximized between the feature values, which leads to poor recognition rate.

To overcome the above-mentioned drawbacks, an unsupervised algorithm (mLBP-LPQ-MLR) implemented for enhancing the performance of age estimation.

\section{Proposed system}

This paper proposes an innovative age estimation method based on feature level fusion of histogram based local texture descriptors. This method consists of 3 modules: 1) Database Collection and Feature Extraction using mLBP and LPQ 2) Feature level fusion 3) Aging function modelling with multiple 
linear regression. These modules explained in the following sub-sections.

\subsection{Feature extraction using MLBP}

LBP is a powerful method for describing image texture by thresholding the surrounding pixels with a centre pixel [9-11]. The LBP method has been widely used in many researches such as age estimation [911], gender recognition, finger vein recognition, facial expression recognition, and face recognition. The main advantage of LBP method is that it offers the texture descriptor robustness to the variations of illumination and rotation. Besides, the fast processing can done by LBP method. The LBP operator [11] defined as Eq. (1).

$$
\begin{aligned}
& L B P_{R, P}=\sum_{i=0}^{P-1} s\left(g_{i}-g_{c}\right)^{2^{i}}, \\
& \text { where } s(x)=\left\{\begin{array}{l}
1, i f x \geq 0 \\
0, \text { if } x<0
\end{array}\right\}
\end{aligned}
$$

Where, $P$ is the number of neighbouring pixels, $R$ is the radius of the LBP circle (the distance from the centre pixel to the neighbouring pixels), $g_{i}$ and $g_{c}$ are the grey level of neighbouring pixels and centre pixel, respectively, and $s(x)$ is the threshold function. Varying the values of $R$ and $P$, then extract image texture features at different scales and resolutions [11]. Originally, the LBP operator makes the texture descriptor by using a $3 \times 3$ mask. In this case, the values of $R$ and $P$ are 1 and 8 , respectively. According to the human's age, the features for age estimation appear in different size (bigger/smaller spot, wrinkle, etc.). In addition, the resolution and size of facial image also causes the size difference of the features. Consequently, varying the values of $R$ and $P$ makes our method obtain the sufficient information of features at different scales and resolution. Fig. 1 shows an example of texture feature of a facial image at different values of $R$ and $P$. As shown in Fig. 1, while the LBP operator with smaller $R$ and $P$ values extracts the fine textures of narrow thickness (Fig. 1) that with the bigger values helps our method to extract the coarse textures of wide thickness.

The extracted LBP binary codes from Eq. (1), are classified into uniform or non-uniform patterns, as shown in Fig. 2 [11]. As the human becomes older, several facial texture features such as wrinkle and spot appears [9-11]. Based on this characteristic, our research uses the LBP operator to describe the image texture and extract the feature for age estimation. A uniform pattern contains at most two bitwise transitions from 0 to 1 (or 1 to 0 ), as shown in Fig. 2. Otherwise, a pattern classified as non-uniform. Uniform patterns are useful for describing image textures, such as spots, corners, and lines, whereas non-uniform patterns contain insufficient information for describing image texture. Fig. 3 shows the examples of representing the textures of spot, corner, and edge into the uniform patterns of 0 , 3, and 4 of Fig. 2, respectively.
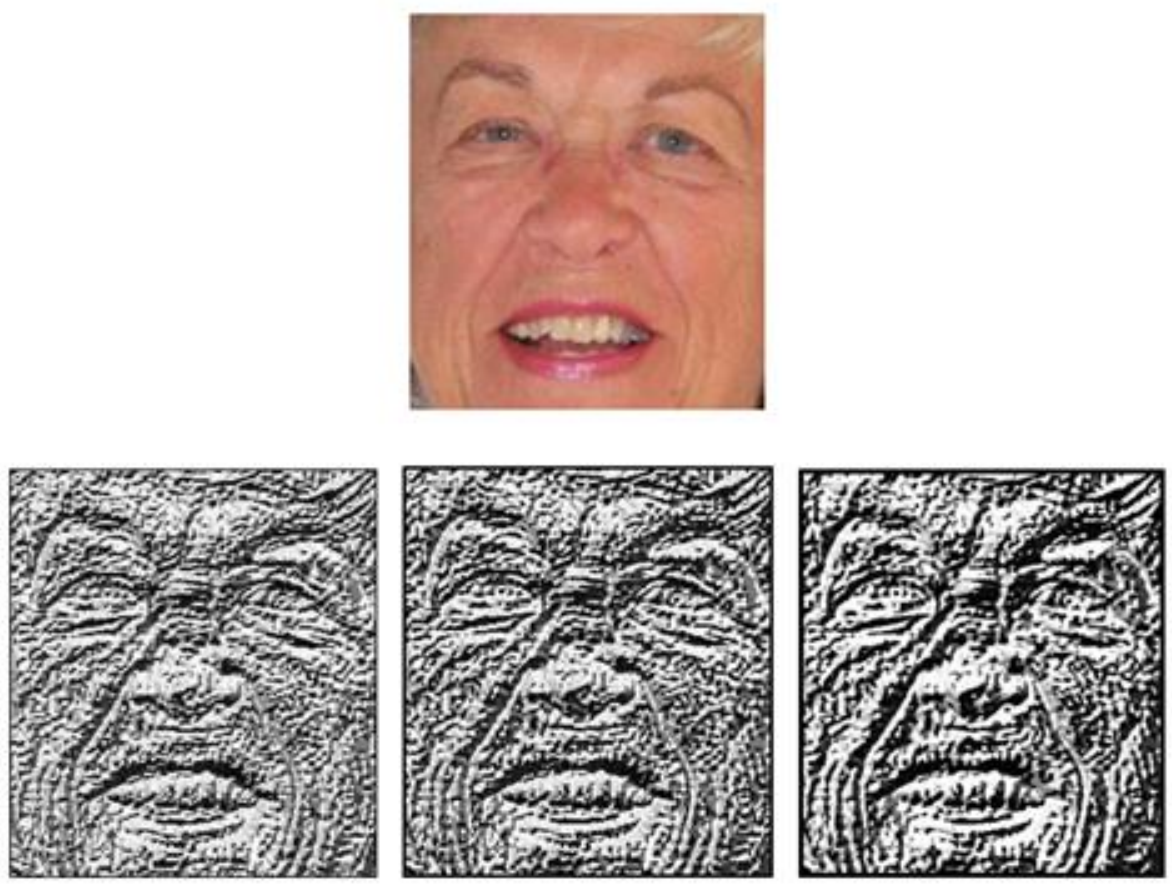

Figure. 1 An example of LBP texture feature extraction 

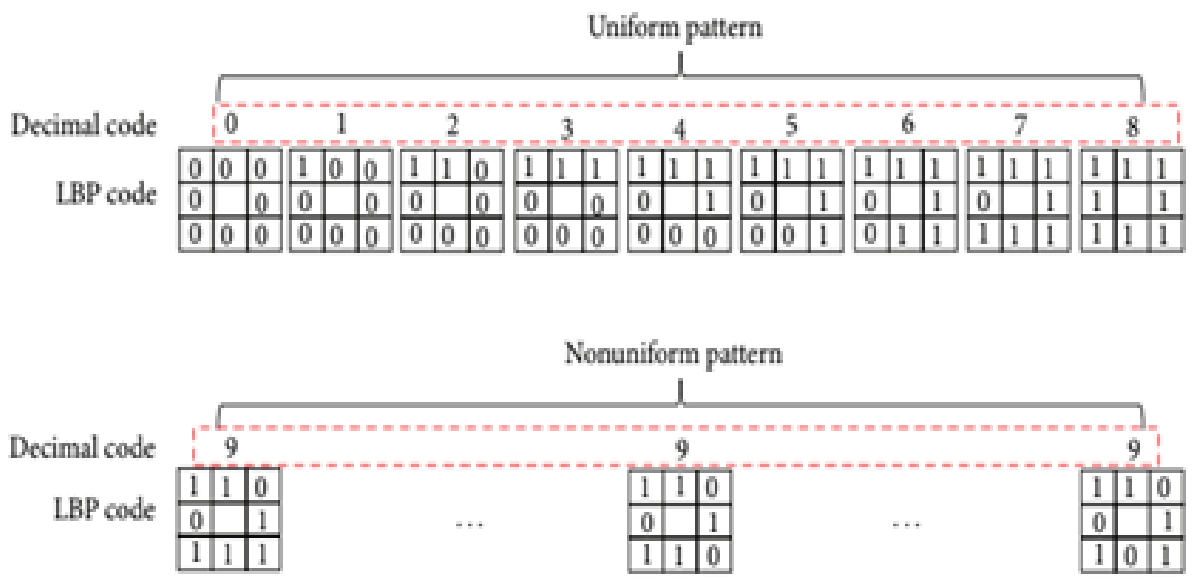

Figure. 2 Example of uniform and non-uniform patterns and their assigned decimal codes in the case in which and are one and eight

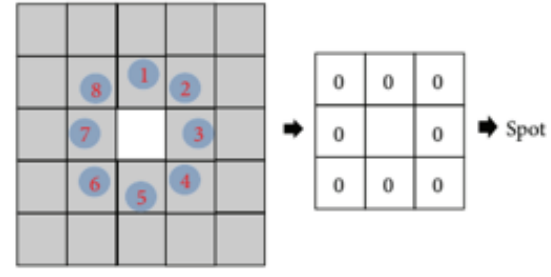

(a)

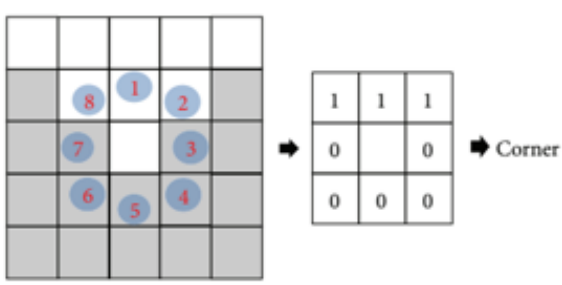

(b)

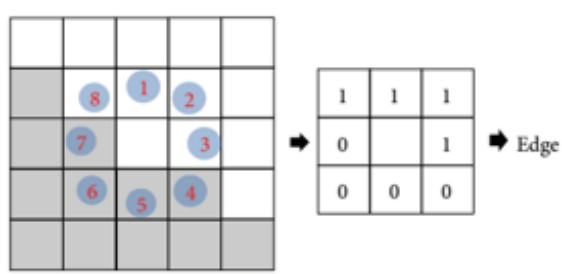

(c)

Figure. 3 Examples of uniform patterns in describing the texture features: (a) spot feature, (b) corner feature, and (c) edge feature

Then, our system forms the image descriptor by accumulating the LBP code histogram (uniform or non-uniform code) over the texture. Fig. 2 shows an example of uniform and non-uniform codes as well as the assigned codes in the case that $P$ and $R$ are one and eight, respectively. Uniform codes assigned decimals from zero to eight, whereas all of the nonuniform codes assigned the decimal nine. By accumulating the histogram of assigned decimal codes of uniform and non-uniform codes in an image, the histogram of texture appearance used as the image descriptor in age estimation [9-11].

The LBP code histogram represents the distribution of image textures in an image. The LBP features of binary code can represent the more local texture compared to the histogram features. However, the LBP binary code can be much affected by image misalignment, and use the histogram features in the research. To extract the various features of image texture, the proposed system divides the input image into local sub-blocks and forms the descriptor (feature vector) for each sub-block. Consequently, the final descriptor of the image formed by concatenating the descriptor of each sub-block. Fig. 4 depicts the method for constructing the LBP descriptor of an image. The feature vectors of each sub-block concatenated together as shown in Fig. 4.

In our research, the order of concatenating the feature vectors of sub-blocks is from left to right and up to down. That is, the histogram of the upper-left sub-block is included first and that of lower right one is included last in the final LBP feature vector. Based on the single-level LBP method of Fig. 4, our method constructs the MLBP features by concatenating the several feature vectors of the single-level LBP, as shown in Fig. 5. The accuracy of age estimation by LBP is affected by the size of sub-block. With the larger sub-block, the global features extracted by LBP whereas with the smaller one, the local features can be obtained. In order to extract the various features globally and locally, use the mLBP of Fig. 5 instead of single-level LBP of Fig. 4. The optimal level (with which the smallest error of age estimation obtained) is determined experimentally to be three for mLBP [11]. 


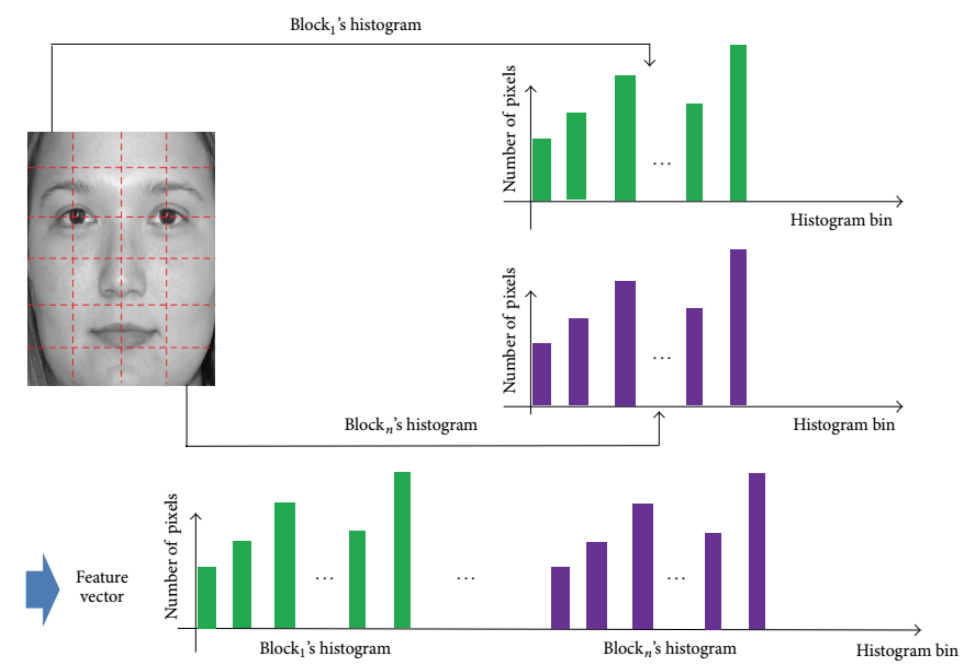

Figure. 4 Method for constructing the LBP feature vector from multiple sub-blocks
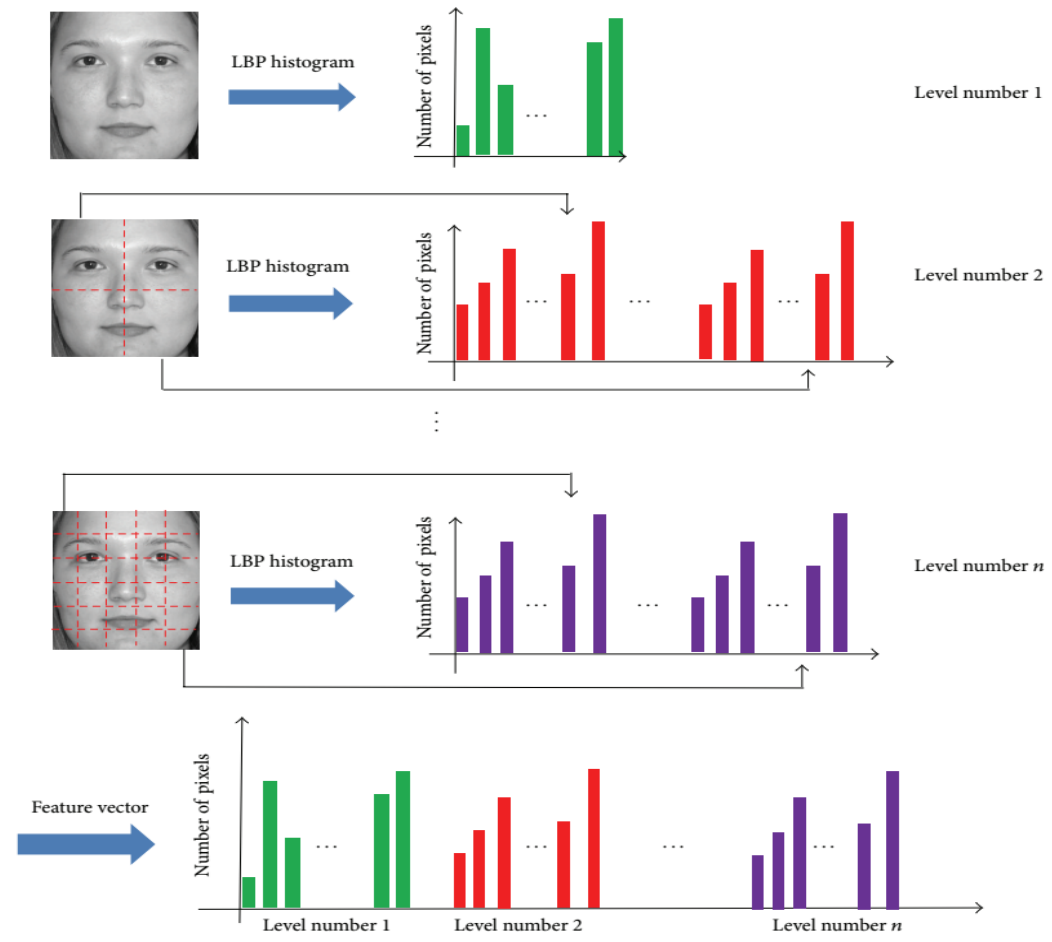

Figure. 5 Method for constructing MLBP feature vector from multiple single-level LBP feature vectors

\subsection{Local phase quantization}

LPQ is a blur insensitive texture descriptor based on the blur invariance property of the Fourier phase spectrum [12]. In this method, LPQ codes computed in local image windows using discrete Fourier transform (DFT) and the results presented as a histogram. The discrete model for spatially invariant blurring of an original image $f(x)$ can expressed by a convolution as $g(x)=(f \times h)(x)$, where $g(x)$ is the observed image $h(x)$ is the point spread function (PSF) of the blur. In the Fourier domain this corresponds to $G(u)=F(u) \cdot H(u)$, where $G(u)$,
$F(u)$ and $H(u)$, the DFTs of the blurred image $g(x)$, the original image $f(x)$, and the PSF $h(x)$, respectively. If the magnitude and phase parts separated, it denoted as $|G(u)|=$ $|F(u)| .|H(u)|$ and $\angle G(u)=\angle F(u)+\angle H(u)$. If we assume that the blur PSF $h(x)$, is centrally symmetric, namely $h(x)=h(-x)$, its Fourier transform is always real valued given by Eq. (2).

$$
\angle H(u)=\left\{\begin{array}{c}
0 \text { if } H(u) \leq 0 \\
\pi \text { if } H(u)<0
\end{array}\right\}
$$

Consequently, the phase of the observed image $\angle G(u)$ is invariant to centrally symmetric blur, at the 
frequencies. The phase of observed image mathematically denoted in the Eq. (3).

$$
\angle G(u)=\angle F(u) \text { for all } \angle H(u) \geq 0
$$

Where $H(u)$ is positive.

Let $N_{x}$ defines the M-by-M neighbourhood around the pixel position $x$ of the image $f(x)$. The twodimensional (2-D) DFT or, more precisely, short term Fourier transform (STFT) of this image window defined by Eq. (4).

$$
F(u, x)=\sum_{y \in N_{x}} f(x-y) e^{-j 2 \pi u^{T} y}=w_{u}^{T} f_{x}
$$

Where, $w_{u}$ is the basis vector of the 2-D DFT at frequency $u$, and $f_{x}$ is the vector containing all M2 pixels in $N_{x}$. Using 2-D convolutions $f(x) e^{-j 2 \pi u^{T} x}$ for all $u$ is the efficient way of STFT implementation. As the basis functions are separable, this 2-D computation can perform by using 1-D convolutions for the rows and columns successively. Only the complex coefficients of $u_{1}=[a, 0]^{T}, u_{2}=[0, a]^{T}, u_{3}=[a, a]^{T}, u_{4}=[a,-a]^{T}$, where, $a$ is a scalar frequency. Below the first zero crossing of $H(u)$ that satisfies, the Eq. (3) considered in LPQ. For each pixel position, this results in a vector given by Eq. (5) and Eq. (6).

$$
\begin{aligned}
& F_{x}^{c}=\left[F\left(u_{1}, x\right), F\left(u_{2}, x\right), F\left(u_{3}, x\right), F\left(u_{4}, x\right)\right. \\
& F_{x}=\left[\operatorname{Re}\left\{F_{x}^{c}\right\}, \operatorname{Im}\left\{F_{x}^{c}\right\}\right]^{T}
\end{aligned}
$$

Where, $\operatorname{Re}\{$.$\} and \operatorname{Im}\{$.$\} return real and$ imaginary parts of a complex number, respectively. The corresponding 8-by- $M^{2}$ transformation matrix presented in Eq. (7).

$$
\begin{aligned}
& W= \\
& {\left[R e\left\{w_{u 1}, w_{u 2}, w_{u 3}, w_{u 4}\right\}, \operatorname{Im}\left\{w_{u 1}, w_{u 2}, w_{u 3}, w_{u 4}\right\}\right.}
\end{aligned}
$$

So, that $F_{x}=w f_{x}$. Next, $G_{x}$ is computed for all image positions, i.e., $x \in\left\{x_{1}, 2, \ldots x_{N},\right\}$ and the resulting vectors are quantized using a simple scalar quantizer $q_{j}$ represented in Eq. (8).

$$
q_{j}=\left\{\begin{array}{c}
1, i \text { if } q_{j} \geq 0 \\
0, \text { if otherwise }
\end{array}\right\}
$$

Where, $q_{j}$ is the $j^{\text {th }}$ component of $G_{x}$. The quantized coefficients represented as integer values between 0 and 255 using binary coding presented in Eq. (9).

$$
b=\sum_{j=1}^{8} q_{j} 2^{j-1}
$$

Finally, the histogram of these integer values used as a feature vector.

\subsubsection{Feature fusion}

In biometrics, feature level, score level and decision level fusion used to combine multiple features [14]. The features are combined using feature level fusion contains richer information about the raw data [13]. For this reason, feature level fusion is adopted in the study to combine the local features produced using various texture descriptors.

The mLBP and LPQ features of a given image extracted in the feature extraction step. Then feature normalization performed using $\mathrm{z}$-score normalization in Eq. (10),

$$
\hat{f}_{i}=\frac{f_{i}-\mu_{i}}{\sigma_{i}}, i=1,2,3
$$

Where, $f_{i}$ is the $i^{\text {th }}$ feature vector, $\mu_{i}$ is the mean and $\sigma_{i}$ is the standard deviation of the $i^{\text {th }}$ feature vector and $f_{l}$ is the normalized feature vector. If the normalized feature vectors donated by $\hat{f}_{m L B P}$ and $\hat{f}_{L P Q}$, the fused feature vector $f$ is the concatenation of these normalized features as follows in Eq. (11).

$$
f=\left[\hat{f}_{m L B P}, \hat{f}_{L P Q}\right]
$$

\subsubsection{Age estimation}

In this study age estimation performed using MLR [15]. MLR is a method used to model the linear relationship between a dependent variable and one or more independent variables. MLR based on least squares: the model is fit such that the sum-of-squares of differences of observed and predicted values is minimized. The model expresses the value of a dependent variable as a linear function of one or more independent variables and an error term as follows in Eq. (12).

$$
y=b_{0}+b_{1} x_{1}+b_{2} x_{2}+\cdots+b_{n} x_{n}=e
$$

Where, $b_{0}$ the regression is constant, $x_{n}$ is the value of nth predictor, $b_{n}$ is the coefficient on the $n^{\text {th }}$ predictor, $n$ is the total number of predictors, $y$ is the predicted and $e$ is the error term. The model in (11) estimated by least squares, which yields parameter estimates such that the sum of squares of errors minimized. Taking the corresponding fused features as the aging features, the following equation formulates the fitted age estimation model. For each 
feature vector $x_{i}$ in training set with $M$ samples represented in Eq. (13).

$$
\left\{y_{i}=b_{0}+b_{1} x_{i, 1}+b_{2} x_{i, 2}+\cdots+b_{n} x_{i, n}\right\}_{i=1}^{M}
$$

Where, $y_{i}$ is the estimated age $x_{i}, n$ is $n^{\text {th }}$ value of the $i^{\text {th }}$ feature vector, $b_{n}$ is the estimated coefficient on $n^{\text {th }}$ value of feature vector and $b_{0}$ is the estimated regression constant.

\section{Experimental results and discussion}

The age classification experiment performed on the FG-NET Aging Database [6] and MORPH database [16] which are the popular databases in the face age estimation used by the research community. The FG-NET Aging database contains 1002 highresolution colour or grayscale face images from 82 subjects ranging from age 0 to 69 . Images in the database display facial appearance changes in pose, illumination, expression, etc. In addition, there are only few images of persons older than 40 in the database. Table 1, shows the age range distribution of the images that used in the FG-NET experiment. The MORPH Database contains more than 55000 images of more than 13000 individuals ranging from age 16 to 77 . The average number of images per individuals is 4. For MORPH experiment, use 20 randomly selected samples for each age value, which range from age 16 to 65 .

In FG-NET experiment, for each sample in dataset, the age class values labelled according to the exact age information. The age class scheme, which illustrated in Table 1. In all samples of a single person are used as the testing set and the remaining samples are used as the training set. For comparison purposes, Mean Absolute Error (MAE) used, which is the most commonly used metric for age estimation. Table 2, shows the MAE of age estimation for different kinds of features which are used as face image feature vectors for the age classifiers. It can observed in Table 2 that, using all textural features in combination with the geometric features, contributes positively to the performance of age estimation. M. Kilinc, and Y.S. Akgul, [6] achieves 5.05 MAE on the FG-NET Aging Database.

As previously mentioned, the images in the FGNET Database, not equally distributed over age ranges. For a detailed analysis of the age estimation method, calculated the MAE for each decade separately. The comparative results of the MAEs per decade (MAE/D) for different kinds of features showed in Table 3.

As previously mentioned, overlapping age groups performs better with our interpolation method than the non-overlapping age groups. In order to verify this, test the proposed method with non-overlapping age class scheme. The age is partitioned into seven different classes such that Class A (0-3), Class B (47), Class C (8-17), Class D (18-29), Class E (30-40), Class F (41-55), Class G (56-70), Class H (70+). The samples are assigned one group label. Our best MAE for all age groups obtained using the fusion of $\mathrm{mLBP}$ and LPQ features as expected. The experimental results showed in the last column of Table 4.

The age class scheme is used in FG-NET experiment is not adequate for MORPH experiment, because the face image dataset that is used in MORPH experiment does not contain samples for age values which range from 0 to 15 . Then, for each classifier, Leave-One-Out evaluation scheme is used. In each fold, one sample used as the testing set and the remaining samples are used as the training set. Table 5, shows the MAE of age estimation on MORPH Database. In Table 5, the combination of mLBP and LPQ features achieves between MAE on MORPH database compared to the existing approaches [6] and [16].

Table 1. The age range distribution of the images in the FG-NET Database

\begin{tabular}{|c|c|}
\hline Age Class & Number of samples \\
\hline$(0-3)$ & 141 \\
\hline$(3-5)$ & 120 \\
\hline$(4-7)$ & 156 \\
\hline$(6-11)$ & 201 \\
\hline$(8-17)$ & 321 \\
\hline$(12-25)$ & 361 \\
\hline$(18-29)$ & 210 \\
\hline$(26-35)$ & 100 \\
\hline$(30-40)$ & 88 \\
\hline$(36-45)$ & 55 \\
\hline$(41-55)$ & 49 \\
\hline$(46-60)$ & 27 \\
\hline$(56-75)$ & 9 \\
\hline
\end{tabular}

Table 2. MAE of age estimation on FG-NET Database

\begin{tabular}{|c|c|}
\hline Age Estimation Method & MAE \\
\hline Classifier 1 (Geometric - No Cross Ratio) & 7.86 \\
\hline Classifier 2 (Geometric) & 6.68 \\
\hline Classifier 3 (Gabor) & 10.24 \\
\hline Classifier 4 (Geometric +Gabor) & 9.35 \\
\hline Classifier 5 (LBP) & 8.94 \\
\hline Classifier 6 (Geometric + LBP) & 8.18 \\
\hline $\begin{array}{c}\text { Classifier 7 Local Gabor Binary Pattern } \\
\text { (LGBP) }\end{array}$ & 9.55 \\
\hline Classifier 8 (Geometric + LGBP) & 5.05 \\
\hline Proposed MLR+ mLBP + LPQ & 4.85 \\
\hline
\end{tabular}


Table 3. MAE/D of age estimation on FG-NET Database

\begin{tabular}{|c|c|c|c|}
\hline $\begin{array}{c}\text { Age } \\
\text { Ranges }\end{array}$ & $\begin{array}{c}|c| \\
\text { Feature Set } \\
\text { LGBP } \\
\text { (Over Lap) } \\
{[6]}\end{array}$ & $\begin{array}{c}\text { Geometric + } \\
\text { LGBP } \\
\text { (Non-Over } \\
\text { Lap) [6] }\end{array}$ & $\begin{array}{c}\text { Proposed } \\
\text { System }\end{array}$ \\
\hline$(0-10)$ & 3.34 & 5.16 & 3.25 \\
\hline$(11-20)$ & 3.28 & 6.1 & 3.18 \\
\hline$(21-30)$ & 7.17 & 7.67 & 6.99 \\
\hline$(31-40)$ & 10.25 & 16.75 & 10.11 \\
\hline$(41-50)$ & 13.4 & 16.3 & 12.98 \\
\hline$(51-60)$ & 14.57 & 30.99 & 14.50 \\
\hline$(61-70)$ & 24.81 & 34.1 & 34.0 \\
\hline
\end{tabular}

Table 4. MAE of age estimation on MORPH Database

\begin{tabular}{|c|c|}
\hline Age Estimation Method & MAE \\
\hline Classifier 2 (Geometric) & 15.15 \\
\hline Classifier 3 (Gabor) & 9.73 \\
\hline Classifier 4 (Geometric +Gabor) & 8.11 \\
\hline Classifier 5 (LBP) & 12.33 \\
\hline Classifier 6 (Geometric + LBP) & 10.93 \\
\hline Classifier 7 (LGBP) & 8.58 \\
\hline Classifier 8 (Geometric + LGBP) [6] & 6.28 \\
\hline Proposed MLR+ mLBP + LPQ & 5.25 \\
\hline
\end{tabular}

Table 5. MAE/D of age estimation on MORPH database

\begin{tabular}{|c|c|c|c|c|c|}
\hline Age range & $\begin{array}{c}\text { Geometric + } \\
\text { LGBP } \\
\text { (overlap) [6] }\end{array}$ & $\begin{array}{c}\text { Geometric + } \\
\text { LGBP } \\
\text { (non-overlap) [6] }\end{array}$ & $\begin{array}{c}\text { Hybrid principal } \\
\text { component } \\
\text { analysis and log } \\
\text { gabor filter } \\
\text { (overlap) [16] }\end{array}$ & $\begin{array}{c}\text { Hybrid principal } \\
\text { component } \\
\text { analysis and log } \\
\text { gabor filter (non- } \\
\text { overlap) [16] }\end{array}$ & $\begin{array}{c}\text { Proposed } \\
\text { system } \\
\text { (overlap) }\end{array}$ \\
\hline$(11-20)$ & 11.62 & 9.13 & 10.98 & 3.23 & 10.22 \\
\hline$(21-30)$ & 8.04 & 6.5 & 8.73 & 2.56 & 7.0 \\
\hline$(31-40)$ & 7.57 & 5.34 & 8.07 & 4.13 & 5.28 \\
\hline$(41-50)$ & 8.38 & 7.06 & 7.77 & 4.82 & 6.65 \\
\hline$(51-60)$ & 6.44 & 5.23 & 5.98 & 2.90 & 5.20 \\
\hline$(61-70)$ & 8.57 & 5.43 & 6.12 & 6.09 & 5.33 \\
\hline
\end{tabular}

Table 6. Comparative analysis

\begin{tabular}{|c|c|c|c|}
\hline \multirow{2}{*}{ References } & \multicolumn{3}{|c|}{ Feature Set } \\
\cline { 2 - 4 } & $\begin{array}{c}\text { FG- } \\
\text { NET }\end{array}$ & MORPH & Description \\
\hline Lai [5] & 4.88 & 8.03 & $\begin{array}{c}\text { Distribution } \\
\text { Based age group } \\
\text { estimation }\end{array}$ \\
\hline Kilinc [6] & 5.05 & 6.28 & $\begin{array}{c}\text { Age Group } \\
\text { Based } \\
\text { estimation }\end{array}$ \\
\hline Li [7] & 7.01 & 8.81 & $\begin{array}{c}\text { Layer Based } \\
\text { Estimation }\end{array}$ \\
\hline Lu [8] & 4.36 & 4.37 & $\begin{array}{c}\text { Expression } \\
\text { Based } \\
\text { Estimation }\end{array}$ \\
\hline $\begin{array}{c}\text { S.K } \\
\text { Gangadharaiah, } \\
\text { and H.N. } \\
\text { Suresh [16] }\end{array}$ & - & 6.97 & $\begin{array}{c}\text { Pose variation } \\
\text { based estimation }\end{array}$ \\
\hline $\begin{array}{c}\text { Proposed } \\
\text { Dropos }\end{array}$ & 4.85 & 5.25 & $\begin{array}{c}\text { Age Group } \\
\text { based estimation }\end{array}$ \\
\hline
\end{tabular}

For a detailed analysis of the age estimation method, calculate the MAE for each decade separately for the MORPH database. The comparative results of the MAEs per decade (MAE/D) for different kinds of features showed in Table 6 . It clearly shows our method is better than the current year research.

\section{Conclusion}

In this paper, a new age estimation method developed for investigating the performance of different histogram-based local texture descriptors. In this method, multi-level LBP and LPQ texture descriptors used to extract holistic and spatial age related features. In spatial feature extraction, the age estimation accuracy of discrete-block and proposed overlapped age block removal improves the MAE. Then, feature fusion performed to investigate the age estimation accuracy of different combinations of local texture descriptors. Using this scheme, MAE's 4.85 and 5.25 years obtained for FG-NET, MORPH databases, respectively. In addition, the proposed approach improves the estimation accuracy regardless of the texture descriptors and databases. In future, appropriate descriptor level features combined with a multi objective classifier for classifying the overlapped age groups.

\section{References}

[1] A. Hadid, M. Pietikäinen, and T. Ahonen, "A discriminative feature space for detecting and recognizing faces", In: Proc. of International Conf. on Computer Vision and Pattern Recognition (CVPR'04), pp.797-804, 2014. 
[2] M. Albert, K. Ricanek, and E. Patterson, "A review of the literature on the aging adult implications for forensic science research and applications", Forensic Sci Int, Vol.172, No.1, pp.1-9, 2007.

[3] M. Gonzalez-Ulloa and E.S. Flores, "Senility of the face-basic study to understand its causes and effects", Plastics \& Reconstructive Surgery, Vol.36, No.2, pp.239-246, 1965.

[4] S. Kumar, S. Ranjitha, and H. N. Suresh, "An Active Age Estimation of Facial image using Anthropometric Model and Fast ICA", Journal of Engineering Science \& Technology Review, Vol.10, No.1, pp.100-106, 2017.

[5] D. Lai, Y. Chen, X. Luo, J. Du, and T. Wang, "Age estimation with dynamic age range", Multimedia Tools and Applications, Vol.76, No.5, pp.6551-73, 2016.

[6] M. Kilinc and Y.S. Akgul, "Automatic human age estimation using overlapped age groups", In: Proc. of International Conf. On Computer Vision, Imaging and Computer Graphics, Theory and Application, pp.313-325, 2013.

[7] Y. Li, Z. Peng, D. Liang, H. Chang, and Z. Cai, "Facial age estimation by using stacked feature composition and selection", The Visual Computer, Vol.32, No.12, pp.1525-1536, 2016.

[8] J. Lu, V.E. Liong, and J. Zhou, "Cost-sensitive local binary feature learning for facial age estimation", IEEE Transactions on Image Processing, Vol.24, No.12, pp.5356-5368, 2015.

[9] S.E. Choi, Y.J. Lee, S.J. Lee, K.R. Park, and J. Kim, "Age estimation using a hierarchical classifer based on global and local facial features," Pattern Recognition, Vol.44, No.6, pp.1262-1281, 2011.
[10] A. Gunay and V.V. Nablyev, "Automatic age classifcation " with LBP," In: Proc. of $23^{\text {rd }}$ International Conf. On Computer and Information Sciences (ISCIS '08), Istanbul, Turkey, pp.1-4, 2008.

[11] D.T. Nguyen, S.R. Cho, and K.R. Park, "Human age estimation based on multi-level local binary pattern and regression method," In: Proc. of International Conf. On Future Information Technology, Vol.309, pp.433-438, 2014.

[12] V. Ojansivu and J. Heikkila, "Blur insensitive texture classification using local phase quantization", In: Proc. of International Conf. On Image and Signal Processing, Vol.5099, pp.236-243, 2008

[13] A. Ross and R. Govindarajan, "Feature level fusion in biometric systems", In: Proc. of International Conf. On Biometric Consortium Conference, pp.1-2, 2004

[14] A. Ross and A. Jain, "Information fusion in biometrics", Pattern Recogn Lett, Vol.24, No.13, pp.2115-2125, 2003.

[15] A. Valenzuela, S. Martin-de las Heras, J.M. Mandojana, J. de Dios Luna, M. Valenzuela, and E. Villanueva, "Multiple regression models for age estimation by assessment of morphologic dental changes according to teeth source", The American journal of forensic medicine and pathology, Vol.23, No.4, pp.386389, 2002.

[16] S.K. Gangadharaiah and H.N. Suresh, "Overlapped Semantic Age Group Estimation Using Hybrid PCA and Log Gabor Filter", International Journal of Intelligent Engineering and Systems, Vol.11, No.2, pp.1-9, 2018. 